\title{
THE DISTRIBUTION OF RADEMACHER SUMS
}

\author{
S. J. MONTGOMERY-SMITH
}

(Communicated by William D. Sudderth)

Abstract. We find upper and lower bounds for $\operatorname{Pr}\left(\sum \pm x_{n} \geq t\right)$, where $x_{1}$, $x_{2}, \ldots$ are real numbers. We express the answer in terms of the $K$-interpolation norm from the theory of interpolation of Banach spaces.

\section{INTRODUCTION}

Throughout this paper, we let $\varepsilon_{1}, \varepsilon_{2}, \ldots$ be independent Bernoulli random variables (that is, $\operatorname{Pr}\left(\varepsilon_{n}=1\right)=\operatorname{Pr}\left(\varepsilon_{n}=-1\right)=\frac{1}{2}$ ). We are going to look for upper and lower bounds for $\operatorname{Pr}\left(\sum \varepsilon_{n} x_{n}>t\right)$, where $x_{1}, x_{2}, \ldots$ is a sequence of real numbers such that $x=\left(x_{n}\right)_{n=1}^{\infty} \in l_{2}$.

Our first upper bound is well known (see, for example, Chapter II, §59 of [5]):

$$
\operatorname{Pr}\left(\sum \varepsilon_{n} x_{n}>t\|x\|_{2}\right) \leq e^{-t^{2} / 2}
$$

However, if $\|x\|_{1}<\infty$, this cannot also provide a good lower bound, because then we have another upper bound:

$$
\operatorname{Pr}\left(\sum \varepsilon_{n} x_{n}>\|x\|_{1}\right)=0 .
$$

To look for lower bounds, we might first consider using some version of the central limit theorem. For example, using Theorem 7.1.4 of [2], it can be shown that for some constant $c$ we have

$$
\left|\operatorname{Pr}\left(\sum \varepsilon_{n} x_{n}>t\|x\|_{2}\right)-\frac{1}{\sqrt{2} \pi} \int_{t}^{\infty} e^{s^{2} / 2} d s\right| \leq c\left(\frac{\|x\|_{3}}{\|x\|_{2}}\right)^{3} .
$$

Thus, for some constant $c$ we have that if $r \leq c^{-1}\left(\log \|x\|_{3} /\|x\|_{2}\right)^{1 / 2}$, then

$$
\operatorname{Pr}\left(\sum \varepsilon_{n} x_{n}>t\|x\|_{2}\right) \geq c^{-1} \int_{t}^{\infty} e^{-s^{2} / 2} d s \geq \frac{c^{-2} e^{-t^{2} / 2}}{t} .
$$

Received by the editors December 22, 1988 and, in revised form, August 30, 1989.

1980 Mathematics Subject Classification (1985 Revision). Primary 60C05; Secondary 60G50.

Key words and phrases. Rademacher sum, Holmstedt's formula. 
However, we should hope for far more. From (1) and (2), we could conjecture something like

$$
\operatorname{Pr}\left(\sum \varepsilon_{n} x_{n}>c^{-1} \inf \left\{\|x\|_{1}, t\|x\|_{2}\right\}\right) \geq c^{-1} e^{-c t^{2}} .
$$

Actually such a conjecture is unreasonable-one should not take infimums of norms, but instead one should consider the following quantity:

$$
\begin{aligned}
K\left(x, t ; l_{1}, l_{2}\right) & =K_{1,2}(x, t) \\
& =\inf \left\{\left\|x^{\prime}\right\|_{1}+t\left\|x^{\prime \prime}\right\|_{2}: x^{\prime}, x^{\prime \prime} \in l_{2}, x^{\prime}+x^{\prime \prime}=x\right\} .
\end{aligned}
$$

This norm is well known to the theory of interpolation of Banach spaces (see, for example [1] or [3]). For small $t$, this norm looks a lot like $t\|x\|_{2}$, but as $t$ gets much larger, it starts to look more like $\|x\|_{1}$. In fact, there is a rather nice approximate formula due to T. Holmstedt (Theorem 4.1 of [3]): if we write $\left(x_{n}^{*}\right)_{n=1}^{\infty}$ for the sequence $\left(\left|x_{n}\right|\right)_{n=1}^{\infty}$ rearranged into decreasing order, then

$$
c^{-1} K_{1,2}(x, t) \leq \sum_{n=1}^{\left\lfloor t^{2}\right\rfloor} x_{n}^{*}+t\left(\sum_{n=\left\lfloor t^{2}\right\rfloor+1}^{\infty}\left(x_{n}^{*}\right)^{2}\right)^{\frac{1}{2}} \leq K_{1,2}(x, t),
$$

where $c$ is a universal constant.

In this paper, we will prove the following result.

Theorem. There is a constant $c$ such that for all $x \in l_{2}$ and $t>0$ we have

$$
\operatorname{Pr}\left(\sum \varepsilon_{n} x_{n}>K_{1,2}(x, t)\right) \leq e^{-t^{2} / 2}
$$

and

$$
\operatorname{Pr}\left(\sum \varepsilon_{n} x_{n}>c^{-1} K_{1,2}(x, t)\right) \geq c^{-1} e^{-c t^{2}} .
$$

An interesting example is $x=\left(n^{-1}\right)_{n=1}^{\infty}$. Then $c^{-1} \log t \leq K_{1,2}(x, t) \leq$ $c \log t$, and hence

$$
c^{-1} \exp (-\exp (c t)) \leq \operatorname{Pr}\left(\sum \varepsilon_{n} n^{-1}>t\right) \leq c \exp \left(-\exp \left(c^{-1} t\right)\right) .
$$

This is quite different behavior than that which we might have expected from the central limit theorem.

We might also consider $x=\left(n^{-1 / p}\right)_{n=1}^{\infty}$, where $1<p<2$. This example leads us to deduce Proposition 2.1 of [7]. More involved methods allow us to rederive the results of [8] (which include the above-mentioned result from [7]). We do not go into details.

We also deduce from the following corollary.

Corollary. There is a constant $c$ such that for all $x \in l_{2}$ and $0<t \leq\|x\|_{2} /\|x\|_{\infty}$ we have

$$
\operatorname{Pr}\left(\sum \varepsilon_{n} x_{n}>c^{-1} t\|x\|_{2}\right) \geq c^{-1} e^{-c t^{2}}
$$


Proof. It is sufficient to show that there is a constant $c$ such that if $0<t \leq$ $\|x\|_{2} /\|x\|_{\infty}$, then

$$
K_{1,2}(x, t) \leq t\|x\|_{2} \leq c K_{1,2}(x, t) .
$$

The left-hand inequality follows straight away from the definition of $K_{1,2}(x, t)$. The right-hand side follows easily from Holmstedt's formula; obviously if $t<$ 1 , and otherwise because

$$
\sum_{n=1}^{\left\lfloor t^{2}\right\rfloor} x_{n}^{*} \geq\left\lfloor t^{2}\right\rfloor \frac{\|x\|_{2}}{t} \geq \frac{t}{2}\|x\|_{2} .
$$

\section{Proof of THEOREM}

In order to prove the theorem, we will need some new norms on $l_{2}$, and a few lemmas.

Definition. For $x \in l_{2}$ and $t>0$, define the norm

$$
J\left(x, t ; l_{\infty}, l_{2}\right)=J_{\infty, 2}(x, t)=\max \left\{\|x\|_{\infty}, t\|x\|_{2}\right\} \text {. }
$$

Lemma 1. For $t>0$, the spaces $\left(l_{2}, K_{1,2},(\cdot, t)\right)$ and $\left(l_{2}, J_{\infty, 2}\left(\cdot, t^{-1}\right)\right)$ are dual to one another, that is, for $x \in l_{2}$ we have

$$
K_{1,2}(x, t)=\sup \left\{\sum x_{n} y_{n}: y \in l_{2}, J_{\infty, 2}\left(y, t^{-1}\right) \leq 1\right\} .
$$

Proof. This is elementary (see, for example Chapter 3, Exercise 1-6 of [1]).

Definition. For $x \in l_{2}$ and $t \in \mathbf{N}$, define the norm

$$
\|x\|_{P(t)}=\sup \left\{\sum_{m=1}^{t}\left(\sum_{n \in B_{m}}\left|x_{n}\right|^{2}\right)^{1 / 2}\right\},
$$

where the supremum is taken over all disjoint subsets, $B_{1}, B_{2}, \ldots, B_{t} \subseteq \mathbf{N}$.

Lemma 2. If $x \in l_{2}$ and $t^{2} \in \mathbf{N}$, then

$$
\|x\|_{P\left(t^{2}\right)} \leq K_{1,2}(x, t) \leq \sqrt{2}\|x\|_{P\left(t^{2}\right)} .
$$

Proof. To show the first inequality, note that we have

$$
\|x\|_{P\left(t^{2}\right)} \leq\|x\|_{1} \text { and }\|x\|_{P\left(t^{2}\right)} \leq t\|x\|_{2} \text {. }
$$

Hence

$$
\begin{aligned}
K_{1,2}(x, t) & =\inf \left\{\left\|x^{\prime}\right\|_{1}+t\left\|x^{\prime \prime}\right\|_{2}: x^{\prime}+x^{\prime \prime}=x\right\} \\
& \geq \inf \left\{\left\|x^{\prime}\right\|_{P\left(t^{2}\right)}+\left\|x^{\prime \prime}\right\|_{P\left(t^{2}\right)}: x^{\prime}+x^{\prime \prime}=x\right\} \\
& \geq\|x\|_{P\left(t^{2}\right)},
\end{aligned}
$$

where the last step follows by the triangle inequality. 
For the second inequality, we start by using Lemma 1 . For any $\delta>0$, let $y \in l_{2}$ be such that

$$
(1-\delta) K_{1,2}(x, t) \leq \sum x_{n} y_{n} \text { and } J_{\infty, 2}\left(y, t^{-1}\right)=1 .
$$

Now pick numbers $n_{0}, n_{1}, n_{2}, \ldots, n_{t^{2}} \in\{0,1,2, \ldots, \infty\}$ by induction as follows: given $0=n_{0}<n_{1}<\cdots<n_{m}$, let

$$
n_{m+1}=1+\sup \left\{\nu: \sum_{n=n_{m}+1}^{\nu}\left|y_{n}\right|^{2} \leq 1\right\} .
$$

Since $\|y\|_{\infty} \leq 1$, we have that $\sum_{n=n_{m}+1}^{n_{m+1}}\left|y_{n}\right|^{2} \leq 2$. Also, as $\|y\|_{2} \leq t$, it follows that $n_{t^{2}}=\infty$. Therefore

$$
\begin{aligned}
(1-\delta) K_{1,2}(x, t) & \leq \sum x_{n} y_{n} \\
& \leq \sum_{m=1}^{t^{2}}\left(\sum_{n=n_{m-1}+1}^{n_{m}}\left|y_{n}\right|^{2}\right)^{1 / 2}\left(\sum_{n=n_{m-1}+1}^{n_{m}}\left|x_{n}\right|^{2}\right)^{1 / 2} \\
& \leq \sqrt{2}\|x\|_{P\left(t^{2}\right)} .
\end{aligned}
$$

Since this is true for all $\delta>0$, the result follows.

The following lemma is due to Paley and Zygmund.

Lemma 3. If $x \in l_{2}$, then given $0<\lambda<1$ we have

$$
\operatorname{Pr}\left(\sum \varepsilon_{n} x_{n}>\lambda\|x\|_{2}\right) \geq \frac{1}{3}\left(1-\lambda^{2}\right)^{2} .
$$

Proof. See Chapter 3, Theorem 3 of [4].

$N$. ow we proceed with the proof of the theorem. First we will show that

$$
\operatorname{Pr}\left(\sum \varepsilon_{n} x_{n}>K_{1,2}(x, t)\right) \leq e^{-t^{2} / 2} \text {. }
$$

Given $\delta>0$, let $x^{\prime}, x^{\prime \prime} \in l_{2}$ be such that $x^{\prime}+x^{\prime \prime}=x$, and

$$
(1+\delta) K_{1,2}(x, t)>\left\|x^{\prime}\right\|_{1}+t\left\|x^{\prime \prime}\right\|_{2} .
$$

Then

$$
\begin{aligned}
\operatorname{Pr}\left(\sum \varepsilon_{n} x_{n}>(1+\delta) K_{1,2}(x, t)\right) \leq & \operatorname{Pr}\left(\sum \varepsilon_{n} x_{n}^{\prime}>\left\|x^{\prime}\right\|_{1}\right) \\
& +\operatorname{Pr}\left(\sum \varepsilon_{n} x_{n}^{\prime \prime}>t\left\|x^{\prime \prime}\right\|_{2}\right) \\
\leq & 0+e^{-t^{2} / 2},
\end{aligned}
$$

where the last inequality follows from equations (1) and (2) above. Letting $\delta \rightarrow 0$, the result follows.

Now we show that for some constant $c$ we have

$$
\operatorname{Pr}\left(\sum \varepsilon_{n} x_{n}>c^{-1} K_{1,2}(x, t)\right) \geq c^{-1} e^{-c t^{2}} .
$$


First let us assume that $t^{2} \in \mathbf{N}$. Given $\delta>0$, let $B_{1}, B_{2}, \ldots, B_{t^{2}} \subseteq \mathbf{N}$ be disjoint subsets such that $\bigcup_{m=1}^{t^{2}} B_{m}=\mathbf{N}$ and

$$
\|x\|_{P\left(t^{2}\right)} \leq(1+\delta) \sum_{m=1}^{t^{2}}\left(\sum_{n \in B_{m}}\left|x_{n}\right|^{2}\right)^{1 / 2} .
$$

Then

$$
\begin{gathered}
\operatorname{Pr}\left(\sum \varepsilon_{n} x_{n}>\frac{1}{2} K_{1,2}(x, t)\right) \geq \operatorname{Pr}\left(\sum \varepsilon_{n} x_{n}>\frac{1}{\sqrt{2}}\|x\|_{P\left(t^{2}\right)}\right) \\
\geq \operatorname{Pr}\left(\sum_{m=1}^{t^{2}} \sum_{n \in B_{m}} \varepsilon_{n} x_{n} \geq \frac{1}{\sqrt{2}}(1+\delta) \sum_{m=1}^{t^{2}}\left(\sum_{n \in B_{m}}\left|x_{n}\right|^{2}\right)^{1 / 2}\right) \\
\geq \prod_{m=1}^{t^{2}} \operatorname{Pr}\left(\sum_{n \in B_{m}} \varepsilon_{n} x_{n} \geq \frac{1}{\sqrt{2}}(1+\delta)\left(\sum_{n \in B_{m}}\left|x_{n}\right|^{2}\right)^{1 / 2}\right) \\
\geq\left(\frac{1}{3}\left(1-\frac{1}{2}(1+\delta)^{2}\right)^{2}\right)^{t^{2}},
\end{gathered}
$$

where the last step is from Lemma 3. If we let $\delta \rightarrow 0$, then we see that

$$
\operatorname{Pr}\left(\sum \varepsilon_{n} x_{n}>\frac{1}{2} K_{1,2}(x, t)\right) \geq \exp \left(-(\log 12) t^{2}\right) .
$$

This proves the result for $t^{2} \in \mathbf{N}$. For $t \geq 1$, note that

$$
K_{1,2}(x, t) \leq K_{1,2}(x\lceil t\rceil) \text { and }\lceil t\rceil^{2} \leq 4 t^{2},
$$

and hence the result follows (with $c=4 \log 12$ ). For $t<1$, the result may be deduced straightaway from Holmstedt's formula and Lemma 3.

\section{ACKNOWLEDGMENTS}

All the work in this paper appears in my Ph.D. thesis [6], taken at Cambridge University under the supervision of Dr. D. J. H. Garling, to whom I would like to express my thanks. I would also like to express my gratitude to the Science and Engineering Research Council, who financed my studies in Cambridge.

\section{REFERENCES}

1. C. Bennett and R. Sharpley, Interpolation of operators, Academic Press, New York, 1988.

2. K. L. Chung, A course in probability theory, 2nd. ed., Academic Press, New York, 1974.

3. T. Holmstedt, Interpolation of quasi-normed spaces, Math. Scand. 26 (1970), 177-199.

4. J.-P. Kahane, Some random series of functions, Cambridge Stud. Adv. Math. 5, 1985.

5. P.-A. Meyer, Martingales and stochastic integrals I, Springer-Verlag, Berlin, 284, 1972. 
6. S. J. Montgomery-Smith, The cotype of operators from $C(K)$, Ph.D. thesis, Cambridge, August 1988.

7. G. Pisier, De nouvelles caractérisations des ensembles de Sidon, Mathematical analysis and applications, Adv. Math. Suppl. Stud. 7B (1981), 686-725.

8. V. A. Rodin and E. M. Semyonov, Rademacher series in symmetric spaces, Analyse Math. 1 (1975), 207-222.

Department of Mathematics, University of Missouri at Columbia, Columbia, Missouri 65211 